\title{
REPRESENTATIONS OF COMPLEX SEMISIMPLE LIE GROUPS AND LIE ALGEBRAS ${ }^{1}$
}

\author{
BY K. R. PARTHASARATHY, R. RANGA RAO AND V. S. VARADARAJAN
}

Communicated by J. W. Green, January 4, 1966

1. Notation. The object of this note is to announce some results on representations of complex semisimple Lie groups and Lie algebras.

(S) is a semisimple Lie algebra over $C$, the field of complex numbers. (S), considered over $R$, the field of real numbers, is denoted by $\$_{0}$.

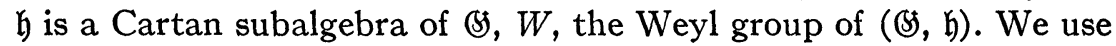
the standard terminology in the theory of semisimple Lie algebras (Jacobson [3] and Harish-Chandra [2(a)], [2(b)], [2(c)]). $P_{0}$ is a positive system of roots, fixed once for all and $S_{0}=\left\{\alpha_{1}, \cdots, \alpha_{l}\right\}$, the associated fundamental system, $\mathfrak{n}=\sum_{\alpha \in P_{0}}(5)-\alpha ; \mathfrak{n}$, considered as a Lie algebra over $R$, is denoted by $\mathfrak{n}_{0} \cdot \mathfrak{h}_{0}=\sum_{\alpha} R \cdot H_{\alpha}$.

Fix a square root $(-1)^{1 / 2}$ of -1 in $C$. $\mathfrak{l}_{0}$ is a compact form of $(5)$ containing $(-1)^{1 / 2} \mathfrak{h}_{0} . \mathfrak{S}_{0}=\mathfrak{l}_{0}+\mathfrak{h}_{0}+\mathfrak{n}_{0}$ is an Iwasawa decomposition of $\mathbb{B}_{0}$ and $G=K \cdot A_{+} \cdot N$ the corresponding decomposition of $G$. $c\left(X \rightarrow X^{c}\right)$ is the conjugation of $\&$ corresponding to the compact form $\mathfrak{P}_{0}$. Let $\hat{\$}$ denote the Lie algebra $\$ \times(\$)$ over $C$, and let

$$
\text { i: } X \rightarrow\left(X^{c}, X\right) \quad(X \in(\mathfrak{)}) \text {. }
$$

$(\hat{\mathbb{S}}, i)$ is a complexification of $\mathbb{B J}_{0}$. For any $X \in \mathbb{B}$ let $\bar{X}=(X, X)$,

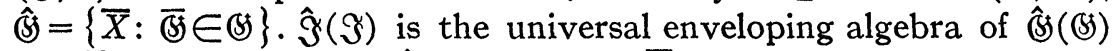
and $\bar{\Im}$ the subalgebra of $\hat{\Im}$ generated by $\bar{\S}$. For any dominant integral $\lambda, \mu \in \mathfrak{h}^{*} \pi_{\lambda}$ denotes the associated irreducible representation of (5) and $\pi_{\lambda}^{-}$that of $\overline{G S}$ (under the isomorphism $\left.X \rightarrow \bar{X}\right) ; \pi(\lambda, \mu)$ is the irreducible representation $\pi_{\lambda} \times \pi_{\mu}$ of $\hat{\xi}$ (Kronecker product).

2. A theorem on finite dimensional representations. We have:

THEOREM 1. Let $\lambda, \mu \in \mathfrak{h}^{*}$ be dominant integral, $\nu=\lambda-\mu^{*}$ and $\nu^{0}$ the unique dominant integral element in the orbit $w \cdot \nu$. Then the representation $\pi_{\nu^{0}}$ of $\overline{\text { ङS }}$ occurs exactly once in the restriction of $\pi(\lambda, \mu)$ to $\overline{\$}$.

3. The homomorphisms $h_{Q}$. For $X \in(S)$ and $a \in \Im$ we write $[X, a]=X a-a X . a$ is said to be of rank 0 if $[H, a]=0$ for all $H \in \mathfrak{h}$. Let $\mathfrak{X}$ be the subalgebra of $\Im$ generated by $\mathfrak{h}$. Suppose $Q$ is any positive system of roots. Then, for any $a \in \mathfrak{S}$ of rank 0 , there is a unique $\S_{Q}(a)$ in $\mathfrak{X}$ such that $a \equiv \S_{Q}(a) \bmod \sum_{\alpha \in Q} \Im_{\mathfrak{S}^{\alpha}} \cdot a \rightarrow \beta_{Q}(a)$ is a homo-

1 The present work was done during 1963-1965 when the authors were at the Indian Statistical Institute, Calcutta. 
morphism (of the algebra of all zero rank elements) onto $\mathfrak{X}$. We identify $\mathfrak{X}$ with $P\left(\mathfrak{h}^{*}\right)$ in a natural fashion.

Let $\Omega$ be the centralizer of $\overline{\mathscr{S}}$ in $\hat{\Im}$. Given $\omega \in \Omega$ we can find unique elements $\mathfrak{x}(0)$ and $\mathfrak{x}(\boldsymbol{k})$ of rank 0 in $\Im$ such that

$$
\omega \equiv \mathfrak{x}(0)^{-}+\sum_{k>0} \mathfrak{x}(k)^{-\cdot} \cdot\left(H_{\alpha_{1}}\right)^{k_{1}} \cdots\left(H_{\alpha_{l}}\right)^{k_{l}}
$$

modulo $\hat{\Im} \hat{\mathfrak{n}}$, where $\hat{\mathfrak{n}}=\boldsymbol{C} \cdot\left(\boldsymbol{i} \cdot \mathfrak{n}_{0}\right)$. We define $h_{Q}(\omega ; \cdot, \cdot)$ as the polynomial on $\mathfrak{h}^{*} \times \mathfrak{h} *$ given by

$$
h_{Q}(\omega ; \lambda, \nu)=\zeta_{Q}(\mathfrak{x}(0))(\nu)+\sum \beta_{Q}(\mathfrak{x}(k))(\nu) \lambda\left(H_{\alpha_{1}}\right)^{k_{1}} \cdots \lambda\left(H_{\alpha_{l}}\right)^{k_{l} l} .
$$

$\omega \rightarrow h_{Q}(\omega ; \cdot, \cdot)$ can then be proved to be a homomorphism of $\Omega$ into $P\left(\mathfrak{h}^{*} \times \mathfrak{h}^{*}\right)$. If $V$ is the space on which $\pi(\lambda, \mu)$ of Theorem 1 acts and if $V_{0}$ is the subspace transforming according to $\pi_{\nu^{0}}$ under $\pi(\lambda, \mu)(\bar{\Im})$, then for $\omega \in \Omega$ and $v \in V_{0}, \pi(\lambda, \mu)(\omega) v=h_{Q}(\omega ; \lambda, \nu) v$ where $Q$ is a positive system in which $\nu$ is dominant.

Let $2 \delta=\sum_{\alpha \in P_{0}} \alpha$ and for $s \in W, \lambda \in \mathfrak{h}^{*}$, let $s^{A} \lambda=s \lambda+s \delta-\delta$.

4. The representations $\hat{\pi}_{\lambda, \nu}$. We shall now define a class of integrable irreducible representations of $\hat{\Im}$ (by integrable we mean that they are the infinitesimal forms of representations of the group $G$; (cf. Harish-Chandra [2(b)], [2(c)]). Let $\nu \in \mathfrak{h}^{*}$ be integral and $\overline{\mathfrak{M}}_{\nu}$ the unique maximal left ideal of $\bar{\Im}$ containing all $\bar{X}_{\beta}, \bar{H}_{\beta}-\nu\left(H_{\beta}\right) \cdot 1$ $(\beta \in Q)$.

Theorem 2. Let $\nu, \lambda \in \mathfrak{h}^{*}, \nu$ integral, and let $Q$ be a positive system under which $\nu$ is dominant. Then there exists a unique maximal left ideal of $\hat{\Im}$ containing $\overline{\mathfrak{M}}_{\nu}$ and $\omega-h_{Q}(\omega ; \lambda, \nu) \cdot 1$ for all $\omega \in \Omega$. The representation $\hat{\pi}_{\lambda, \nu}$ of $\hat{\Im}$ defined by the maximal left ideal is integrable. In the restriction of $\hat{\pi}_{\lambda, \nu}$ to $\bar{\Im}$, the representation $\pi_{\nu^{0}}^{-}$occurs exactly once, and for any dominant integral $\rho \in \mathfrak{h}^{*}, \pi_{\rho}^{-}$cannot occur unless $\nu$ is a weight of $\pi_{\rho}$, in which case, $\pi_{\rho}^{-}$occurs with a multiplicity $\leqq d_{\rho}^{\nu}$ where $d_{\rho}^{\nu}$ is the multiplicity of $\nu$ in $\pi_{\rho}$. If $\lambda$ and $\mu=(\lambda-\nu)^{*}$ are dominant integral, $\hat{\pi}_{\lambda, \nu}$ is equivalent to $\pi(\lambda, \mu)$. Finally, for fixed $\lambda, \nu$, all the representations $\hat{\pi}_{s} A_{\lambda, s \nu}$ are equivalent.

5. The rings $\Re_{\nu}$ for dominant integral $\nu$. Let $\nu$ be dominant integral. Let $\Re_{\nu}$ be the range of the homomorphism $\omega \rightarrow h_{P_{0}}(\omega ; \cdot, \nu)$.

$$
\begin{aligned}
W_{\nu} & =\{s: s \in W, s \cdot \nu=\nu\}, \\
I_{\nu}^{A} & =\left\{p: p \in P\left(\mathfrak{h}^{*}\right), s^{A} \cdot p=p \text { for all } s \in W_{\nu}\right\} .
\end{aligned}
$$

THEOREM 3. $\Re_{\nu} \subseteq I_{\nu}^{A}$. If $\nu=0$ or if $\nu$ is in general position, $\Re_{\nu}=I_{\nu}^{A}$. For general $\nu$, let $\Im_{0}(\nu)$ be defined by 
$\Im_{0}(\nu)=\{a: a \in \Im, a$ is of rank 0 and

$$
\left.\left(\operatorname{ad} X_{\alpha_{i}}\right)^{\eta_{i}+1}(a)=0 ; \text { for } i=1, \cdots, l\right\}
$$

(where $X_{\alpha}$ is a nonzero element of (5) and $\nu_{i}=\nu\left(H_{\alpha_{i}}\right)$ ). Then

$$
\Re_{\nu}=s_{0}^{A} \cdot\left\{\beta_{P_{0}}\left(\Im_{0}\left(-s_{0} \nu\right)\right)\right\} \text {. }
$$

By case considerations we can show that $\Re_{\nu}=I_{\nu}^{A}$ for all $\nu$. But we do not possess a uniform proof of this fact.

6. Representations of class 0 . An integrable representation of $\hat{\Im}$, say $\pi$, is said to be of class 0 if its restriction to $\bar{\Im}$ contains the trivial representation of $\bar{\Im}$. We can show that every integrable irreducible representation of class 0 of $\hat{\Im}$ is equivalent to some $\hat{\pi}_{\lambda, 0}$. We shall say that $\hat{\pi}_{\lambda, 0}$ is complete if for each $\rho$ such that 0 is a weight of $\pi_{\rho}, \pi_{\rho}^{-}$occurs with multiplicity equal to $d_{\rho}^{0}$ in the restriction of $\hat{\pi}_{\lambda, 0}$ to $\bar{\Im}$.

THEOREM 4. For $a \lambda \in \mathfrak{h}^{*}, \hat{\pi}_{\lambda, 0}$ is complete if and only if for each root $\alpha \in P_{0},(\lambda+\delta)\left(H_{\alpha}\right) \notin Z^{*}$ where $Z^{*}$ is the set of nonzero integers.

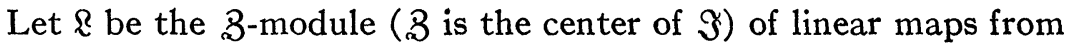
the representation space $V^{\rho}$ of $\pi_{\rho}$ into $\Im$ which intertwine $\pi_{\rho}$ and the adjoint representation, let $d=d_{\rho}^{0}$ and let $\left\{L_{1}, \cdots, L_{d}\right\}$ be a basis of the free module $\&$ (cf. Kostant [4]). Let $\left\{v_{1}, \cdots, v_{d}\right\}$ be a basis of the 0 weight subspace of $V^{\rho}$, and let $K_{\rho}^{\prime}$ be the $d \times d$ matrix of elements of $\mathfrak{X}$ whose $(i-j)$ th element is $\beta_{P_{0}}\left(L_{i} v_{j}\right)$.

TheOREM 5. The multiplicity $m(\rho, \lambda)$ of $\pi_{\rho}^{-}$in $\hat{\pi}_{\lambda, 0}$ is given by

$$
m(\rho, \lambda)=\min \left\{\operatorname{rank} K_{\rho *}^{\prime}(\lambda), \operatorname{rank} K_{\rho}^{\prime}\left(s_{0}^{A} \lambda\right)\right\}
$$

where $s_{0} \in W$ is such that $s_{0} P_{0}=-P_{0}, \rho^{*}=-s_{0} \rho$.

7. Representations of $G$. Let $G$ be the simply connected group corresponding to $\mathfrak{S}_{0}$. Let log denote the inverse of the exponential map from $A_{+}$onto $\mathfrak{h}_{0}$. For any integral $\nu \in \mathfrak{h}^{*}$ let $\psi_{\nu}\left(\exp (-1)^{1 / 2} H_{0}\right)$ $=\exp (-1)^{1 / 2} \cdot \nu\left(H_{0}\right)\left(H_{0} \in \mathfrak{h}_{0}\right) . \psi_{\nu}$ is a character of $M$, the connected component of the centraliser of $A_{+}$in $K$. Let $\mathfrak{S}=\mathbb{R}^{2}(K)$,

$$
\mathfrak{S}(\nu)=\left\{f: f \in \mathfrak{S}, R_{r}(k) f=\psi_{-\nu}(k) f \text { for all } k \in M\right\}
$$

( $\nu$ integral, $R_{r}$ the right regular representation of $K$ ). Following Harish-Chandra [2(b), p. 240] we define, for each $\xi \in \mathfrak{h}^{*}$, the representation $\pi_{\xi, \nu}$ in $\mathfrak{S}(\nu)$ by setting for all $f \in \mathfrak{S}(\nu)$

$$
\left(\pi_{\xi, \nu}(x) f\right)(k)=\exp \left[(\xi+2 \delta)\left(\log a_{+}\left(x^{-1}, k\right)\right)\right] \cdot f\left(\sigma_{x}^{-1}(k)\right)
$$


$(k \in K, x \in G)$; here, for $y \in G$, and $k \in K, y \cdot k=\sigma_{y}(k) \cdot a_{+}(y, k) \cdot n(y, k)$ where $\sigma_{y}(k) \in K, a_{+}(y, k) \in A_{+}, n(y, k) \in N$. $\nu^{0}$ is the dominant integral element in $W \cdot \nu, \mathfrak{S}(\nu)_{\nu 0}$ is the set of all elements in $\mathfrak{S}(\nu)$ which transform under the left regular representations of $K$ according to $\pi_{\nu^{0}}$.

THEOREM 6. The representation $\pi_{\xi, \nu}$ of $G$ in $\mathfrak{S}(\nu)$ is homogeneous. Let $\mathfrak{S}_{(\nu ; \xi)}$ be the smallest closed subspace of $\mathfrak{S}(\nu)$ invariant under $\pi_{\xi, \nu}(G)$ and containing $\mathfrak{S}(\nu)_{\nu 0}$. Then there exists a unique maximal closed subspace $\mathfrak{S}^{\prime}(\nu ; \xi)$ of $\mathfrak{S}(\nu ; \xi)$ invariant under $\pi_{\xi, \nu}(G)$ and not containing $\mathfrak{S}_{(}(\nu)_{\nu_{0}} \mathfrak{S S}^{\prime}(\nu ; \xi)$ is orthogonal to $\mathfrak{S}(\nu)_{\nu 0}$ and the representation of $G$ defined by $\pi_{\xi, \nu}$ in $\mathfrak{S}(\nu ; \xi) / \mathfrak{S g}^{\prime}(\nu ; \xi)$ is irreducible and the associated representation of $\hat{\xi}$ is equivalent to $\hat{\pi}_{\lambda, \nu}$ where $\lambda=\frac{1}{2}(\nu+\xi)-\delta$.

The question as to when the $\pi_{\xi, \nu}$ are themselves irreducible is a crucial one; (cf. Bruhat [1]) for $\nu=0$ we have a complete answer to this question.

Theorem 7. For $\pi_{\xi, 0}$ to be irreducible it is necessary and sufficient that $\frac{1}{2} \xi\left(H_{\alpha}\right) \notin Z^{*}$ for each root $\alpha$. In particular, all unitary representations of the principal nondegenerate series, whose restrictions to $K$ contain the trivial representation of $K$, are irreducible.

Acknowledgement. At an early stage of the present work, the last named author had the opportunity of detailed and highly stimulating discussions with Dr. S. R. S. Varadhan, currently of the Courant Institute of Mathematical Sciences, New York. He would like to record his indebtedness to Dr. Varadhan for those discussions which determined our entire approach to the whole circle of questions outlined in the present work.

\section{BIBLIOGRAPHY}

1. F. Bruhat, Sur les réprésentations induites des groupes de Lie, Bull. Soc. Math. France 84 (1956), 97-205.

2. Harish-Chandra, (a) On some applications of the universal enveloping algebra of a semisimple Lie algebra, Trans. Amer. Math. Soc. 70 (1951), 28-96;

(b) Representations of a semisimple Lie group on a Banach space. I, Trans. Amer. Math. Soc. 75 (1953), 185-243;

(c) Representations of semisimple Lie groups. II, Trans. Amer. Math. Soc. 76 (1954), 26-65.

3. N. Jacobson, Lie algebras, Interscience, New York, 1962.

4. B. Kostant, Lie group representations on polynomial rings, Amer. J. Math. 85 (1963), 327-404.

University of Sheffield, Sheffield

UNIVERSITY OF ILLINOIS AND

University of California, Los Angeles 